\title{
UPDATED MASS PREDICTIONS FROM THE GARVEY-KELSON MASS RELATIONS*
}

\author{
J. JÄNECKE \\ Department of Physics, University of Michigan \\ Ann Arbor, Michigan 48109
}

Part A, Neutron-Rich Nuclides, describes the procedures for estimating masses of neutron-rich nuclei from the transverse Garvey-Kelson mass relation. This relation represents a homogeneous partial difference equation. The most general solutions have been subjected to a $\chi^{2}$-minimization procedure (boundary condition) based on the new atomic mass adjustment of Wapstra and Bos. The resulting solution can be viewed as a many-parameter mass equation with about 500 parameters. About 5000 mass values have been calculated for nuclei with $2 \leq Z \leq 100,4 \leq N \leq 156$, and $N>Z$ (or $N=Z=$ even). The standard deviation between calculated and experimental mass-excess values is $\sigma_{m}=118 \mathrm{keV}$.

Part B, Proton-Rich Nuclides, describes the procedure for estimating masses of proton-rich nuclei with $T \geq 1$ hased on the charge-symmetric mass relation of Kelson and Garvey. Use is made of the experimental Coulomb energy differences between $T=1 / 2$ mirror nuclei $(A \leq 55)$ together with a few estimated values $(57 \leq A \leq 71)$. Mass values have been calculated for about 120 nuclei with $1 \leq T \leq 5 / 2$ and $A \leq 70$. The standard deviation between calculated and experimental mass-excess values is $\sigma_{m} \approx 100 \mathrm{keV}$.

* Supported in part by the U.S. Energy Research and Development Administration, Contract E(111)-2167, and by a research grant from the Michigan Memorial Phoenix Project, University of Michigan 


\title{
CONTENTS
}

\author{
PART A. NEUTRON-RICH NUCLIDES \\ The Garvey-Kelson Relations \\ Method of Mass Prediction \\ PART B. PROTON-RICH NUCLIDES \\ The Kelson-Garvey Relation \\ Method of Mass Prediction
}

\section{PART A. NEUTRON-RICH NUCLIDES}

\section{The Garvey-Kelson Relations}

Nuclidic mass relations were introduced by Garvey and Kelson ${ }^{1,2}$ a number of years ago as a new approach for predicting masses of unknown nuclei from known nuclear masses. The ground-state energy $M(N, Z)$ of a system of $A$ nucleons ( $N$ neutrons and $Z$ protons) is determined uniquely by the nuclear many-body Hamiltonian containing one-, two- and possibly many-body operators. Since the exact form of the Hamiltonian is not known, it is essentially impossible to obtain general solutions of the many-body problem. Instead of introducing models or other assumptions, in this new approach, the question was considered whether the differences $M(N+\Delta N, Z+\Delta Z)-M(N, Z)$ between the masses of neighboring nuclci perhaps can be understood on the basis of more limited knowledge about the nuclear many-body system.

The objective then was to construct a difference equation of the form

$$
\sum_{i=1}^{\alpha} C_{i} M\left(N+\Delta N_{i}, Z+\Delta Z_{i}\right) \approx 0
$$

with $\left|C_{i}\right|=1$ and arbitrary $N$ and $Z$. A proper choice of the coefficients $C_{i}$ hopefully would lead to an approximate cancellation of the neutron and proton singleparticle energies as well as of the interaction energies for all neutron-neutron, proton-proton, and neutron-proton pairs. A necessary condition for such cancellations is that the number of neutrons and protons and of the various pairs cancels in this relation. Nontrivial solutions cxist for $\alpha \geq 6$. For $\alpha=6$ the following two independent solutions were obtained, ${ }^{3,4}$

$$
\begin{aligned}
& M\left(A, T_{z}+2\right)-M\left(A, T_{z}\right) \\
& +M\left(A+1, T_{z}+1 / 2\right)-M\left(A+1, T_{z}+3 / 2\right) \\
& +M\left(A-1, T_{z}+1 / 2\right)-M\left(A-1, T_{z}+3 / 2\right) \approx 0
\end{aligned}
$$

and

$$
\begin{aligned}
& M\left(A+4, T_{z}\right)-M\left(A, T_{z}\right) \\
& \quad+M\left(A+1, T_{z}+1 / 2\right)-M\left(A+3, T_{z}+1 / 2\right) \\
& \quad+M\left(A+1, T_{z}-1 / 2\right)-M\left(A+1, T_{z}-1 / 2\right) \approx 0
\end{aligned}
$$

with $A=N+Z$ and $2 T_{z}=N-Z$. Schematic representations of the transverse and longitudinal relations (2) and (3) are shown in Fig. 1. When the two equations were tested ${ }^{3}$ for all combinations of known nuclear masses, it was found that the deviations from zero are indeed small and essentially random. The standard deviations are on the order of $200 \mathrm{keV}$. Excluded from this test were all combinations which contain nuclei with $N<Z$ or $N=Z=$ odd.

Using an independent-particle picture with fourfold degenerate Hartree-Fock or Nilsson-like single-particle levels, Eqs. (2) and (3) are represented schematically as shown in Fig. 2 (a) and (b). Indeed, single-particle energies as well as the residual interaction energies cancel out in this model if they are assumed to vary slowly with nucleon number $A$. No such cancellation exists if one of the members in Eqs. (2) or (3) is an odd-odd self-conjugate nucleus ( $N=Z=$ odd) as is demonstrated by Fig. 2 (c) and (d). The effective

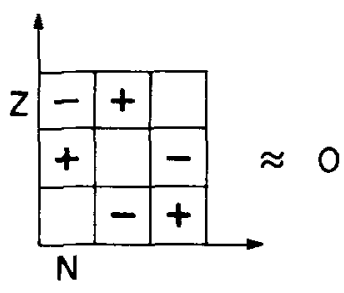

Transverse

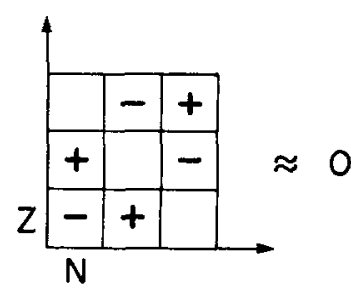

Longitudinal
Fig. 1. Schematic representation of the transverse and longitudinal mass relations (2) and (3). The boxes represent nuclei from the nuclidic chart with $N$ horizontal and $Z$ vertical. The presence of a plus or a minus sign in a box indicates that the mass value of the respective nucleus is to be added or subtracted 
interaction between a neutron and a proton sharing the same space orbital is much stronger than for different orbitals resulting in residuals of up to a few $\mathrm{MeV}$ for the light nuclei (see also Ref. 5).

Simple shell-model mass equations are in accord ${ }^{3,4}$ with Eqs. (2) and (3). If neutrons and protons are assumed to occupy the same $j$-shell, the shell-model expression, which is based on the seniority coupling scheme with isospin as a good quantum number, satisfies Eqs. (2) and (3) exactly. The supermultiplet scheme also yields an expression for the binding energy that is consistent with Eqs. (2) and (3). These expressions further show that deviations are to be expected for $N=Z=$ odd. They result from the dependence on isospin $T$ of the symmetry energy which is not of the form $T^{2}=\left|T_{z}\right|^{2}=1 / 4(N-Z)^{2}$ but instead is approximately of the form $T(T+1)$ which has a cusp at $T=0$.

Equations (2) and (3) are not expected ${ }^{3,4}$ to hold exactly. Due to changes in the nuclear radius or the nuclear shape, small residuals may result from several effects including the variation of single-particle and interaction energies with the number of neutrons and protons. Furthermore, the extreme single-particle picture of Fig. 2 is very simple-minded. The states are generally not angular momentum eigenstates and therefore require configuration mixing, core excitation, etc. Even if the extreme picture is assumed to be valid, small residuals would be expected for odd- $A$ and odd-odd reference nuclei. ${ }^{6}$ These are zero only if the effective neutron-proton interaction is independent of neutron excess or nucleon number, respectively.
Studies of the "fluctuations" of the residuals which appear to be essentially statistical in nature are being carried out by Kelson. ${ }^{7}$ The influence of small systematic effects is being studied by Jänecke et al. ${ }^{6,8}$

\section{Method of Mass Prediction}

Garvey, Kelson, and collaborators ${ }^{1,3}$ have reported two distinctly different methods for predicting masses of neutron-rich and proton-rich nuclei based on Eqs. (2) and (3). First, the equations can be used as recursion relations since they permit an estimate of the unknown mass of one nucleus from the known masses of five adjacent nuclei. This step can then be repeated to obtain estimates for unknown nuclei further away from the line of $\beta$-stability. In a second approach, Eqs. (2) and (3) are treated as homogeneous partial difference equations for the unknown function $M(N, Z)=M\left(A, T_{z}\right)$. The most general solutions have to be obtained and then subjected to boundary conditions. The most general solutions are ${ }^{3,4}$

$$
M(N, Z)=g_{1}(N)+g_{2}(Z)+g_{3}(N+Z)
$$

and

$$
M(N, Z)=f_{1}(N)+f_{2}(Z)+f_{3}(N-Z),
$$

respectively, where $g_{1}, g_{2}, g_{3}, f_{1}, f_{2}$, and $f_{3}$ are arbitrary functions of their arguments. These functions can be constructed essentially uniquely from a $\chi^{2}$-minimization of the differences $M(N, Z)-M_{\exp }(N, Z)$ for all known nuclei with $N>Z$ and $N=Z=$ even. The solutions can be obtained for a wide but not unlimited range of $N$

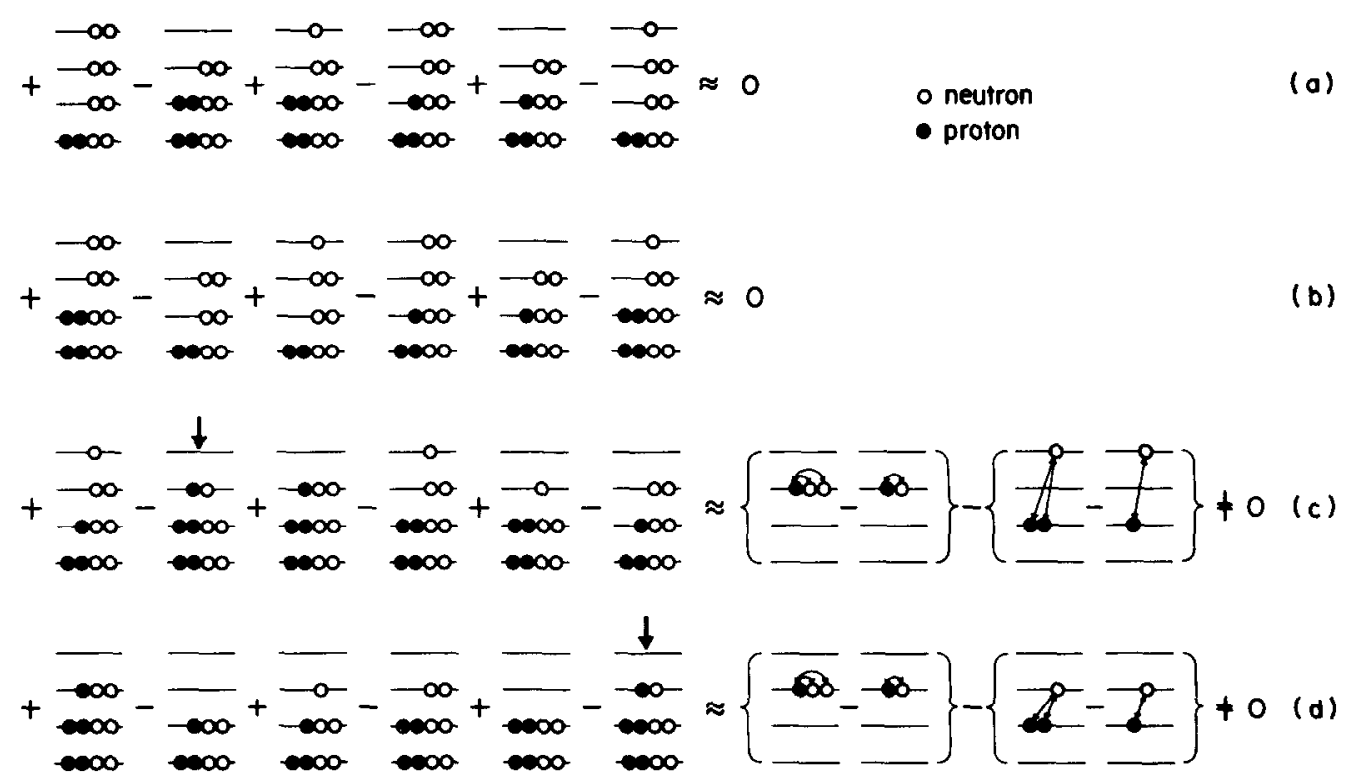

Fig. 2. Representation of the transverse (a) and longitudinal (b) mass relations based on an extreme single-particle picture. The relations are not satisfied [see (c) and (d)] if one of the members is an odd-odd self-conjugate nucleus with $N=Z=$ odd (marked by arrows) 
and $Z$ values. At least two masses must be known for each value of $N$ and $Z$, and at least one for each value of $N+Z$ or $N-Z$. Also, certain trivial functions of the arguments can be added and subtracted to the functions $g_{i}$ and $f_{i}$ without, however, changing the solutions.

Equations (4) and (5) represent two independent solutions. It has been shown earlier ${ }^{3}$ that the two solutions do not in general predict the same values for masses off the stability line. Experimental evidence shows Eq. (4) to agree better with the data. Also, a recent consistency test ${ }^{8}$ for the two solutions showed clearly that Eq. (4) is superior to Eq. (5).

Several years ago Garvey et al. ${ }^{3}$ published functions $g_{1}(N), g_{2}(Z)$, and $g_{3}(N+Z)$ with an extensive listing of predicted mass values. Extensions and revisions have been made more recently ${ }^{9}$ for the region of very light nuclei. Since many new data have become available in the meantime, ${ }^{10}$ it was considered worthwhile to perform a new overall determination of the above functions. Another reason, of course, is that a computer program for solving the inhomogeneous partial difference equation has become available. ${ }^{8}$ The present homogeneous equation represents only a special case of this. The functions $g_{1}(N), g_{2}(Z)$, and $g_{3}(N+Z)$ were obtained from a slightly modified $\chi^{2}$-minimization procedure by solving a system of about 500 linear equations in about 500 unknowns. The new experimental mass values of Wapstra and Bos ${ }^{10}$ were used as input data. By quadratically adding $100 \mathrm{keV}$ to the experimental uncertainties, values with uncertainties of less than $100 \mathrm{keV}$ were given essentially equal weight, and reduced weight was given to those with larger uncertainties. Since the computer program makes use of sparse matrix subroutines, the computing time is about ten seconds on the University of Michigan AMDAHL $470 \mathrm{~V} / 6$ computer and about twice as long on the compatible IBM 370/168.

Instead of Eq. (4), the slightly modified expression

$\Delta M(N, Z)=N \Delta M_{n}+Z \Delta M_{\mathrm{H}}-\alpha A+\beta Z^{2}$

$$
+\eta(N-Z)^{2}+g_{1}(N)+g_{2}(Z)+g_{3}(A)
$$

was used for the calculations. Here, the $\Delta M$ denote mass excesses. Equation (6) is in accord with Eq. (4) since $(N-Z)^{2}=2 N^{2}-2 Z^{2}-(N+Z)^{2}$. The term $\alpha A-\beta Z^{2}-\eta(N-Z)^{2}$ describes very approximately the volume, Coulomb and symmetry energies. The functions $g_{1}(k)$ take on smaller numerical values. The coefficients $\alpha$ and $\beta$ were arbitrarily chosen, and $\eta$ was determined from the condition $\Sigma g_{3}(A)=0$. The functions $g_{1}(N), g_{2}(Z)$, and $g_{3}(A)$ are listed in Table I together with the coefficients $\alpha, \beta$, and $\eta$. They are also displayed in Fig. 3. Equation (6) with Table I allows mass predictions for all nuclei with $4 \leq N \leq 156$, $2 \leq Z \leq 100, \quad 6 \leq A \leq 256, \quad$ and $\quad N>Z$ or
$N=Z=$ even. The standard deviation for differences between calculated and experimental mass excesses is $\sigma_{m}=118 \mathrm{keV}$. About 5000 predicted mass values are included in the tabulation.

\section{PART B. PROTON-RICH NUCLIDES}

\section{The Kelson-Garvey Relation}

Nuclear forces are essentially charge symmetric and also charge independent. The difference in binding energy between conjugate mirror nuclei thus can be ascribed almost entirely to the electrostatic intcraction. Therefore, the unknown mass of a proton-rich nucleus $\left(A,-T_{z}\right)$ can be estimated from the known mass of its neutron-rich mirror nucleus $\left(A,+T_{z}\right)$ provided the Coulomb energy difference between the two nuclei can be estimated,

$$
\begin{aligned}
M\left(A, T_{z}=\right. & -T)-M\left(A, T_{z}=+T\right) \\
= & \Delta E_{c}\left(A, T, T_{z}=-T \mid T_{z}=+T\right) \\
& -2 T_{z}\left(M_{n}-M_{\mathrm{H}}\right) .
\end{aligned}
$$

Here, $A=N+Z$ is the nucleon number and $T_{z}=1 / 2(N-Z)$ is the $z$-component of the isospin $T$. The quantity $\Delta E_{C}$ represents the difference in Coulomb energy in an obvious notation, and $M_{n}-M_{\mathrm{H}}$ is the neutron-hydrogen mass difference. This method is widely used. The Coulomb energies are generally estimated from theoretical or phenomenological considerations or from a combination thereof.

A very simple and successful method has been introduced by Kelson, Garvey, and collaborators. ${ }^{2-4}$ The mass relation

$$
\begin{aligned}
M(A,-1)- & M(A,+1) \\
\approx & M(A-1,-1 / 2)-M(A-1,+1 / 2) \\
& \quad+M(A+1,-1 / 2)-M(A+1,+1 / 2)
\end{aligned}
$$

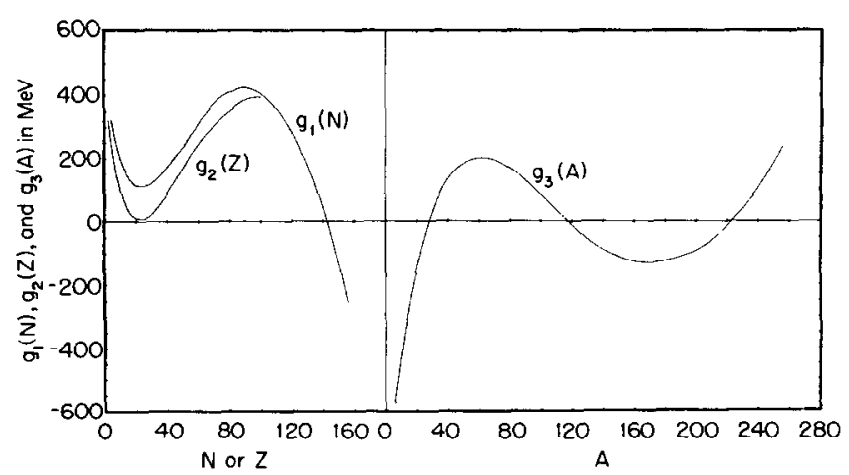

Fig. 3. Plot of the functions $g_{1}(N), g_{2}(Z)$, and $g_{3}(A)$ for the transverse mass equation [Eq. (6)] 
( $A=$ even) connects masses near the $N=Z$ line in a symmetric way. The relation is shown schematically in Fig. 4(a). The approximate validity of the relation can be seen by adopting an independent-particle picture with fourfold degenerate Hartree-Fock or Nilsson-like single-particle levels. Indeed, Fig. 5 shows that all nuclear interactions cancel out under the assumption of charge symmetry of nuclear forces and the assumption that electrostatic interactions between protons are equal on both sides of the equation. The equation

$$
M\left(A, T_{z}=-T\right)-M\left(A, T_{z}=+T\right) \approx \sum_{\substack{A^{\prime}=A-(2 T-1) \\(\text { in steps of two })}}^{A+(2 T-1)}\left[M\left(A^{\prime},-1 / 2\right)-M\left(A^{\prime},+1 / 2\right)\right]
$$

represents a generalized version ${ }^{3,4}$ of Eq. (8). Here, $A=$ even (odd) for $T=$ integer (half-integer). Figure 4(b) shows this relation for $T=5 / 2$ as an example.

$$
\Delta E_{C}(A, T,-T \mid+T) \approx \sum_{\substack{A A^{\prime}=A-(2 T-1) \\ \text { (in steps of two) }}}^{A+(2 T-1)} \Delta E_{C}\left(A^{\prime}, 1 / 2,-1 / 2 \mid+1 / 2\right) .
$$

Equation (9) becomes a Coulomb energy relation (see also Ref. 11) when neutron-hydrogen mass differences are subtracted according to Eq. (7),
Coulomb energy differences between the most protonrich and neutron-rich members of an isospin multiplet with $T \geq 1$ can thus be estimated from the known differences for the $T=1 / 2$ mirror nuclei. Equation (10) follows also from the isobaric multiplet mass equation if certain simple assumptions are made about the linear coefficient.

\section{Method of Mass Prediction}

The masses of $T=1 / 2$ mirror pairs are now known ${ }^{10}$ up to $A=55$, and reliable predictions (estimated error less than $\pm 100 \mathrm{keV}$ ) can be made ${ }^{11}$ a few steps beyond. Also, a great number of neutron-rich nuclei with $T_{z} \geq 1$ are now known in this region. Mass predictions for many proton-rich light nuclei have therefore become possible.

Table II shows the experimental mass differences for $T=1 / 2$ with the calculated and, when available, experimental ${ }^{10}$ mass differences for $T=1,3 / 2,2$, and $5 / 2$. All experimental uncertainties were obtained by quadratically adding the uncertainties for the two respective masses. This represents an overestimate in some cases.

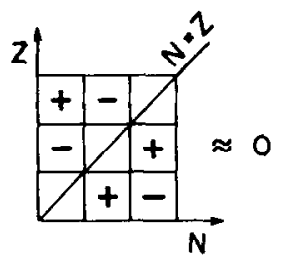

(a)

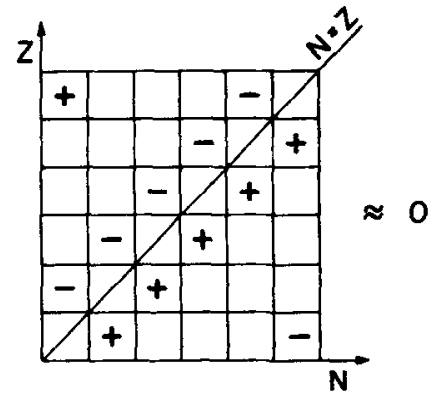

(b)
Fig. 4. Schematic representation of the charge-symmetric KelsonGarvey mass relation [Eq. (3)] for $T=1$ and $T=5 / 2$. The boxes represent nuclei from the nuclidic chart with $N$ horizontal and $Z$ vertical. The presence of a plus or a minus sign in a box indicates that the mass value of the respective nucleus is to be added or subtracted
There exists good agreement between the calculated and the available experimental data. The standard deviation is about $100 \mathrm{keV}$. Systematic deviations of up to a few hundred $\mathrm{keV}$ are also present, as noted earlier, $3,4,12$ when either a proton or a proton pair is unbound (see, for example, $A=4,6,16$ for $T=1$ ). The effect results from a Coulomb perturbation in the wavefunction which leads to an energy shift (ThomasEhrman shift). The true masses for nuclei near and beyond the proton instability line are therefore likely to be slightly lower than predicted. The relevant mass differences which include nuclei for which the predicted proton or two-proton binding energies are negative or less than $200 \mathrm{keV}$ are marked by the dagger symbol $\dagger$ in Table II. Mass predictions for about 120 proton-rich light nuclei with $T \leq 5 / 2$ have become possible with the use of the charge-symmetric Kelson-Garvey mass relation. The predicted mass values are combined with those from Part A from neutron-rich nuclei in the main table of this issue.

\section{Acknowledgments}

Thanks are due to G. T. Garvey and K. T. Hecht for the careful reading of the manuscript and for valuable suggestions. Thanks are due to B. P. Eynon for extensive help with computer programming.

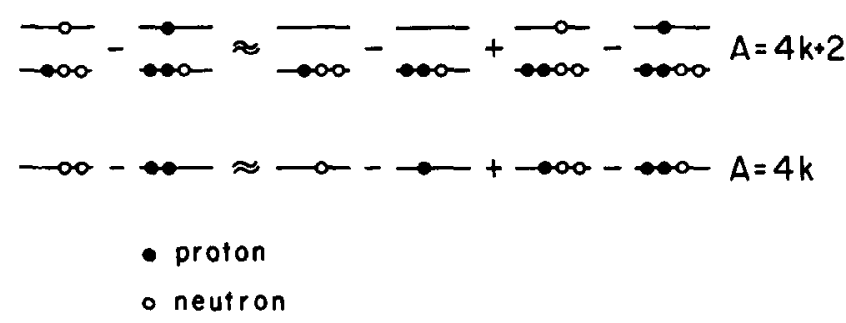

Fig. 5. Representation of the charge-symmetric Kelson-Garvey mass relation [Eq. (3)] for $T=1$ based on an extreme single-particle picture. Filled orbitals below are not shown 


\section{References}

1. G. T. Garvey and I. Kelson, Phys. Rev. Lett. 16, 197 (1966)

2. I. Kelson and G. T. Garvey, Phys. Lett. 23, 689 (1966)

3. G. T. Garvey et al., Rev. Mod. Phys. 41, S1 (1969)

4. G. T. Garvey, Annu. Rev. Nucl. Sci. 19, 433 (1969)

5. W. H. Bassichis and I. Kelson, Phys. Rev. C 5, 1169 (1972)

6. J. Jänecke and H. Behrens, Z. Phys. 256, 236 (1972); Phys. Rev. C 9, 1276 (1974)

7. I. Kelson, in Proceedings of the Fifth International Conference on Atomic Masses and Fundamental Constants, Paris, France, 1975, edited by J. H. Sanders and A. H. Wapstra (Plenum Press, London-
New York, 1975)

8. J. Jänecke and B. P. Eynon, Nucl. Phys. A 243, 326 (1975); in Proceedings of the Fifth International Conference on Atomic Masses and Fundamental Constants, Paris, France, 1975, edited by J. H. Sanders and A. H. Wapstra (Plenum Press, LondonNew York, 1975); ATOMIC Data and Nuclear Data TABLes, this issue

9. C. Thibault and R. Klapisch, Phys. Rev. C 6, 1509 (1972); Phys. Rev. C 9, 793 (1974)

10. A. H. Wapstra and K. Bos, Atomic Data AND Nuclear Data Taki.Fs, this issue

11. J. Jänecke, in Isospin in Nuclear Physics, edited by D. H. Wilkinson (North-Holland, Amsterdam, 1968) p. 355

12. J. Jänecke, Phys. Rev. C 6, 467 (1972) 
TABLE I. Functions $g_{1}(N), g_{2}(Z)$, and $g_{3}(A)$ in $\mathrm{keV}$ for the transverse mass-excess equation

$$
\Delta M(N, Z)=N \Delta M_{n}+Z \Delta M_{\mathrm{H}}-\alpha A+\beta Z^{2}+\eta(N-Z)^{2}+g_{1}(N)+g_{2}(Z)+g_{3}(A)
$$

obtained from a $\chi^{2}$-adjustment to all experimental masses ${ }^{10}$ with $4 \leq N \leq 156,2 \leq Z \leq 100$, and $N>Z$ or $N=Z=$ even. The coefficients are $\alpha=16000 \mathrm{keV}, \beta=120 \mathrm{keV}$, and $\eta=195.8560 \mathrm{keV}$. The mass excesses are $\Delta M_{n}=8071.43 \mathrm{keV}$ and $\Delta M_{\mathrm{H}}=7289.03 \mathrm{keV}$

$g_{1}(N)$

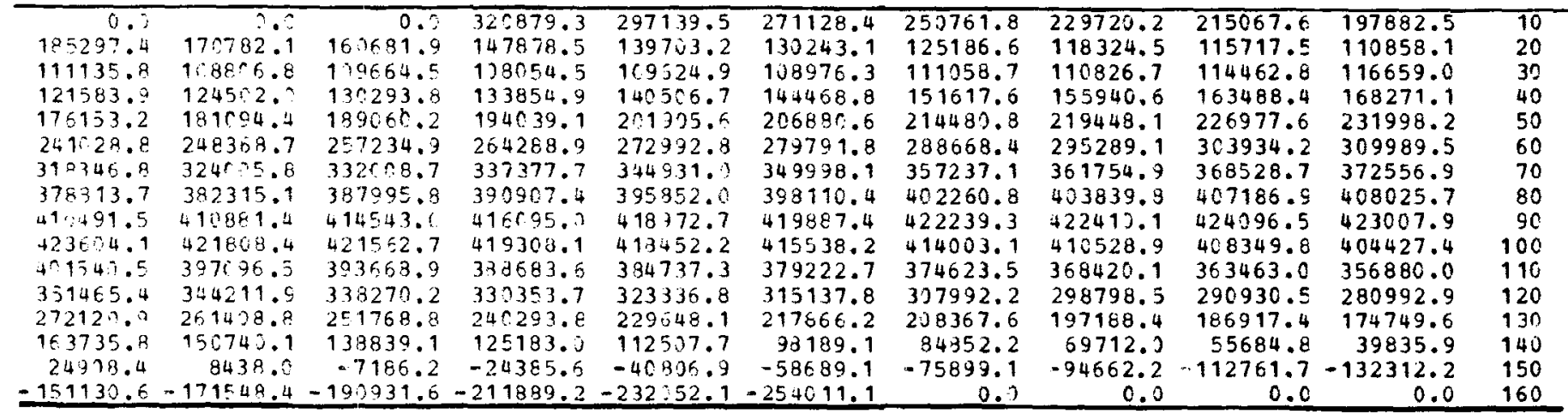

$s_{2}(Z)$

\begin{tabular}{|c|c|c|c|c|c|c|c|c|c|c|}
\hline $\begin{array}{r}9 \\
93454.6 \\
8993.1 \\
27311.2 \\
95651.9 \\
173644.9 \\
25266 \% .8 \\
319337.7 \\
354120.3 \\
396771.8 \\
\end{array}$ & $\begin{array}{r}327879 . ? \\
56390.7 \\
5736.9 \\
31718.0 \\
172132.5 \\
191454.0 \\
258242.1 \\
315619.4 \\
356553.3 \\
387749.6\end{array}$ & $\begin{array}{r}287052.6 \\
54983.2 \\
7035.3 \\
39147.5 \\
11120 \% .8 \\
191139.1 \\
265820.3 \\
391180.2 \\
361929.1 \\
390099.1 \\
\end{array}$ & $\begin{array}{r}249337.7 \\
40810.2 \\
5758.5 \\
44036.7 \\
11748 . .7 \\
198502.6 \\
271033.4 \\
324907.0 \\
365394.7 \\
390644.3\end{array}$ & $\begin{array}{r}217794.0 \\
33119.2 \\
7692.6 \\
51939.4 \\
126496.7 \\
207860.5 \\
278177.6 \\
330172.5 \\
370103.6 \\
392345.3\end{array}$ & $\begin{array}{r}134227.1 \\
24486.0 \\
7571.2 \\
57185.2 \\
132876.0 \\
214735.3 \\
283175.9 \\
333753.8 \\
372748.4 \\
392227.7 \\
\end{array}$ & $\begin{array}{r}160600.3 \\
20012.6 \\
10943.5 \\
65438.3 \\
141940.3 \\
223670.6 \\
289876.9 \\
339154.2 \\
376796.6 \\
393341.2\end{array}$ & $\begin{array}{r}135290.5 \\
14055.9 \\
11979.2 \\
71173.1 \\
148313.6 \\
230088.2 \\
294556.5 \\
342451.6 \\
378780.5 \\
392623.4\end{array}$ & $\begin{array}{r}118009.5 \\
11846.3 \\
17235.7 \\
79859.5 \\
157308.5 \\
238617.6 \\
300848.0 \\
347144.3 \\
382285.2 \\
393116.2\end{array}$ & $\begin{array}{r}97845.8 \\
7634.9 \\
20928.8 \\
86354.2 \\
163554.2 \\
244596.5 \\
305290.9 \\
349875.9 \\
383810.8 \\
399842.6\end{array}$ & $\begin{array}{r}10 \\
20 \\
30 \\
40 \\
50 \\
60 \\
70 \\
80 \\
90 \\
100\end{array}$ \\
\hline
\end{tabular}

$g_{3}(A)$

\begin{tabular}{|c|c|c|c|c|c|c|c|c|c|c|}
\hline $\begin{array}{r}125246.7 \\
111148 . n \\
-90399.3 \\
-56462.0 \\
-9344.8 \\
45567.5 \\
112019.1 \\
194212.4\end{array}$ & 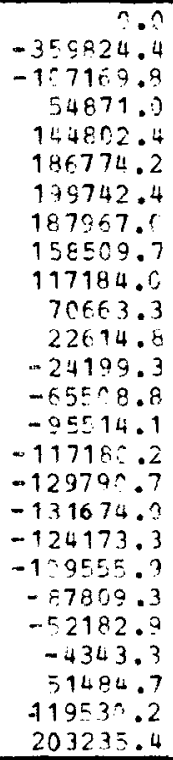 & $\begin{array}{r}56960.2 \\
151115.3 \\
189629.6 \\
193796.8 \\
195955.3 \\
155280.7 \\
112823.5 \\
55940.7 \\
18012.4 \\
-28669.7 \\
-68939.1 \\
-07978.3 \\
-118873.7 \\
-130391.4 \\
-131325.4 \\
-122986.4 \\
-107670.2 \\
-34917.7 \\
-47662.8 \\
993.5 \\
57691.1 \\
127267.7 \\
212516.4\end{array}$ & $\begin{array}{r}13060.4 \\
-33220.2 \\
-72348.5 \\
100561.5 \\
120615.7 \\
13 C 929.2 \\
130988.8 \\
121711.7 \\
106041.0 \\
-82009.3 \\
-43221.6 \\
6171.0 \\
63881.7 \\
135060.1 \\
221924.8\end{array}$ & $\begin{array}{r}88 \\
161 \\
193 \\
198 \\
180 \\
147 \\
103 \\
56 \\
8 \\
-37 \\
-75 \\
-112 \\
-122 \\
-131 \\
-130 \\
-126 \\
-104 \\
-78 \\
-38 \\
11 \\
70 \\
143 \\
231\end{array}$ & $\begin{array}{r}-575288.8 \\
-244315.0 \\
-32982.6 \\
93039.2 \\
165473.3 \\
195338.4 \\
197483.2 \\
177554.0 \\
143534.7 \\
98911.1 \\
51282.9 \\
3515.0 \\
-42024.6 \\
-78774.5 \\
-115246.1 \\
-123645.3 \\
-131733.1 \\
-129686.8 \\
-118952.4 \\
-102267.8 \\
-75597.0 \\
-33974.6 \\
16942.3 \\
76926.6 \\
151158.2 \\
241401.8\end{array}$ & $\begin{array}{r}-536756 \\
-217996 \\
-16188 \\
107649 \\
169811 \\
137001 \\
196619 \\
174792 \\
139438 \\
94280 \\
46619 \\
-915 \\
-46104 \\
-81639 \\
-107337 \\
-124879 \\
-131974 \\
-128875 \\
-117442 \\
-160173 \\
-72209 \\
-29116 \\
22594 \\
83667 \\
159506 \\
0\end{array}$ & $\begin{array}{r}- \\
115 \\
173 \\
197 \\
195 \\
171 \\
134 \\
89 \\
41 \\
-5 \\
-50 \\
-84 \\
-109 \\
-126 \\
-132 \\
-128 \\
-116 \\
-98 \\
-68 \\
-24 \\
28 \\
90 \\
167\end{array}$ & $\begin{array}{l}0.9 \\
3.9 \\
3.5 \\
6.1 \\
0.6 \\
8.6 \\
7.2 \\
6.9 \\
9.4 \\
7.0 \\
6.4 \\
5.1 \\
2.9 \\
7.7 \\
7.2 \\
7.1 \\
C .4 \\
3.9 \\
0.2 \\
1.2 \\
5.4 \\
7.3 \\
4.5 \\
7.7 \\
5.4 \\
0.0\end{array}$ & $\begin{array}{l} \\
-1 \\
-5 \\
-5 \\
-1 \\
-12 \\
-13 \\
-12 \\
-11 \\
-9 \\
-6 \\
-1 \\
10 \\
10\end{array}$ & $\begin{array}{l}200 \\
210 \\
220 \\
230\end{array}$ \\
\hline
\end{tabular}

The first line gives $g_{1}(1), g_{1}(2), \ldots, g_{1}(10)$; the second line gives $g_{1}(11), g_{1}(12), \ldots, g_{1}(20)$, etc. 
TABLE II. Experimental and Calculated Mass Differences between Conjugate Proton-Rich and Neutron-Rich Mirror Nuclei

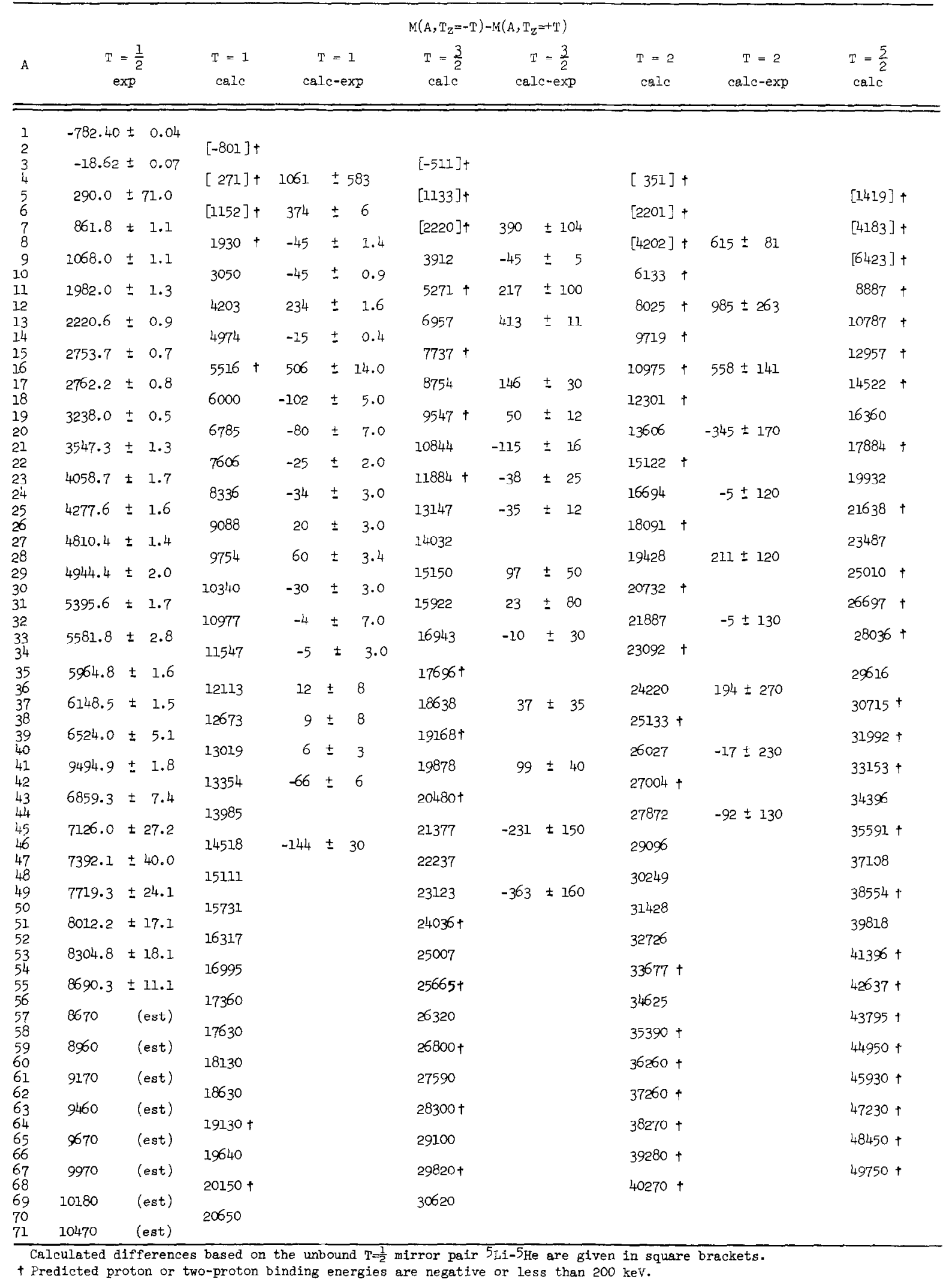

